\title{
COMMENTS
}

Comments are short papers which criticize or correct papers of other authors previously published in the Physical Review. Each Comment should state clearly to which paper it refers and must be accompanied by a brief abstract. The same publication schedule as for regular articles is followed, and page proofs are sent to authors.

\section{Absorbing boundary conditions for inertial random processes}

\author{
Jaume Masoliver and Josep M. Porrà \\ Departament de Física Fonamental, Universitat de Barcelona, Diagonal, 647, 08028-Barcelona, Spain \\ Katja Lindenberg \\ Department of Chemistry and Biochemistry and Institute for Nonlinear Science, University of California, \\ San Diego, La Jolla, California 92093-0340 \\ (Received 1 February 1996)
}

\begin{abstract}
A recent paper by J. Heinrichs [Phys. Rev. E 48, 2397 (1993)] presents analytic expressions for the firstpassage times and the survival probability for a particle moving in a field of random correlated forces. We believe that the analysis there is flawed due to an improper use of boundary conditions. We compare that result, in the white noise limit, with the known exact expression of the mean exit time. [S1063-651X(96)06512-9]

PACS number(s): 02.50.- r, 05.40.+j
\end{abstract}

In two recent works $[1,2]$ Heinrichs has studied some statistical aspects of the motion of a free particle under the influence of a random acceleration modeled as Gaussian noise:

$$
\ddot{X}(t)=F(t),
$$

where $F(t)$ is Ornstein-Uhlenbeck noise, that is, a zero-mean Gaussian process with correlation function

$$
\left\langle F(t) F\left(t^{\prime}\right)\right\rangle=\frac{D}{2 \tau_{c}} e^{-\left|t-t^{\prime}\right| / \tau_{c}},
$$

where $D$ is the noise intensity and $\tau_{c}$ is the correlation time. The main results achieved in Ref. [1] have been to obtain approximate expressions for the joint probability density function, $p(x, v, t)$, of the position $x$ and the velocity $v$, and for the marginal densities $p(x, t)$ and $p(v, t)$. These approximate results, valid for Ornstein-Uhlenbeck driving noise, can be generalized to obtain the exact expressions of $p(x, v, t)$, $p(x, t)$, and $p(v, t)$ for Gaussian driving noises $F(t)$ with arbitrary correlation functions:

$$
\left\langle F(t) F\left(t^{\prime}\right)\right\rangle=k\left(t, t^{\prime}\right) .
$$

Such a generalization has been carried out in Ref. [3].

Heinrichs's second paper on the subject [2] used that approximate expression for the marginal density of the position $p(x, t)$ to deal with the important and difficult problem of obtaining the mean exit time (MET) $T\left(x_{0}\right)$ out of an interval $(0, L)$, for the (initial) position $x_{0}$ of the random particle, regardless of the value of the initial velocity [4]. We first observe that this approach is, at least, incomplete since the MET $T\left(x_{0}, v_{0}\right)$ of an inertial process depends, in a complicated manner, on both the position and the velocity of the particle. Once we know the complete MET $T\left(x_{0}, v_{0}\right)$, the MET $T\left(x_{0}\right)$ is given by the following average over all initial velocities $[5,6]$ :

$$
T\left(x_{0}\right)=\int_{-\infty}^{\infty} p\left(v_{0}\right) T\left(x_{0}, v_{0}\right) d v_{0},
$$

where $p\left(v_{0}\right)$ is the distribution of initial velocities. Heinrichs's derivation of $T\left(x_{0}\right)$ does not involve $p\left(v_{0}\right)$ and, in fact, assumes that $v_{0}=0$. As a consequence, what is evaluated in [2] is the quantity $T\left(x_{0}, v_{0}=0\right) \equiv T\left(x_{0}, 0\right)$.

Moreover, we believe that the evaluation of the mean exit time at zero velocity, $T\left(x_{0}, 0\right)$, is flawed due to the use of improper boundary conditions for the problem. Indeed, in Heinrichs's analysis the mean exit time $T\left(x_{0}\right)$ out of the interval $(0, L)$ is given by

$$
T\left(x_{0}\right)=\int_{0}^{\infty} d t \int_{0}^{L} p\left(x, t \mid x_{0}\right) d x,
$$

where $p\left(x, t \mid x_{0}\right)$ obeys the Fokker-Planck equation

$$
\frac{\partial p}{\partial t}=\frac{1}{2} D_{x}(t) \frac{\partial^{2} p}{\partial x^{2}},
$$

with initial condition

$$
p\left(x, 0 \mid x_{0}\right)=\delta\left(x-x_{0}\right),
$$

and absorbing boundary conditions

$$
p\left(0, t \mid x_{0}\right)=p\left(L, t \mid x_{0}\right)=0 .
$$

Equation (6) is the Fokker-Planck equation for the marginal density of the position if $v_{0}=0$. When $v_{0} \neq 0$ the equation for $p\left(x, v \mid x_{0}, v_{0}\right)$ reads [3] 


$$
\frac{\partial p}{\partial t}=-v_{0} \frac{\partial p}{\partial x}+\frac{1}{2} D_{x}(t) \frac{\partial^{2} p}{\partial x^{2}},
$$

and the marginal density of the position clearly depends on both the initial position $x_{0}$ [cf. Eq. (7)] and the initial velocity $v_{0}$. For a general Gaussian driving noise the function $D_{x}(t)$ is exactly given by [3]

$$
D_{x}(t)=2 \int_{0}^{t} d t^{\prime} \int_{0}^{t^{\prime}} d t^{\prime \prime}\left(2 t-t^{\prime}-t^{\prime \prime}\right) k\left(t^{\prime}, t^{\prime \prime}\right) .
$$

In the case of Ornstein-Uhlenbeck noise this function reads [cf. Eq. (2)]

$$
D_{x}(t)=D t\left[t-\tau_{c}\left(1-e^{-t / \tau_{c}}\right)\right] .
$$

Finally, for Gaussian white noise $\tau_{c} \equiv 0$ and

$$
D_{x}(t)=D t^{2} .
$$

We now show that the boundary conditions (8) are not the appropriate absorbing boundary conditions for an inertial process such as (1). In effect, Eq. (1) represents a two dimensional random process $(X, \dot{X})$, and the proper absorbing boundary conditions for trapping are [7-9]

$$
\begin{array}{ll}
p(L, v, t)=0 \quad \text { if } v \leqslant 0, \\
p(0, v, t)=0 \quad \text { if } v \geqslant 0 .
\end{array}
$$

These are the correct absorbing boundary conditions for the exit problem because the particle has never left the interval and it is impossible to find the particle with positive velocity at $x=0$ or with negative velocity at $x=L[10]$.

Since the marginal density of the position is given by

$$
p(x, t)=\int_{-\infty}^{\infty} p(x, v, t) d v,
$$

we see that

$$
p(L, t)=\int_{0}^{\infty} p(L, v, t) d v
$$

Note that one does not know whether $p(L, v, t)$ is zero or not for all positive values of the velocity. Hence we may not conclude that $p(L, t)=0$. Analogously

$$
p(0, t)=\int_{-\infty}^{0} p(0, v, t) d v
$$

and the assumption $p(0, t)=0$ is also doubtful. We believe that Eq. (8) does not embody the correct absorbing boundary conditions for the random motion of an inertial particle. In fact, Eq. (8) represents absorbing boundary conditions of some first-order diffusion process with a time dependent diffusion coefficient given by Eq. (10).

One might argue that nonetheless these boundary conditions may lead to a good approximation for the mean exit time $T\left(x_{0}, 0\right)$. We can check the goodness of such an approximation by comparing it with the exact result that we have for the case of Gaussian white noise. In effect, when

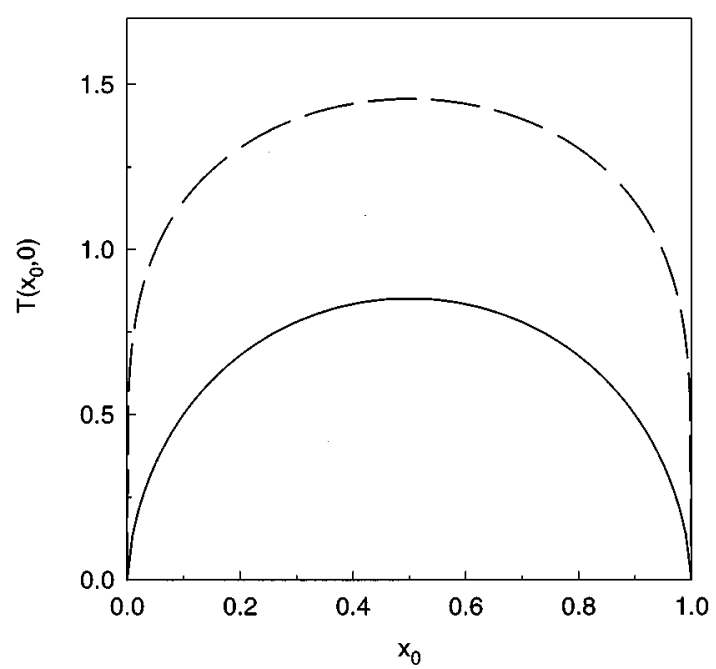

FIG. 1. $T\left(x_{0}, 0\right)$ as a function of $x_{0}$, for noise intensity $D=1$ and interval length $L=1$. The solid curve corresponds to the approximation given by Eq. (19). The dashed curve represents the exact result (15).

$F(t)$ is Gaussian and $\delta$ correlated noise we have recently shown that the exact expression for the mean exit time at zero velocity out of an interval $(0, L)$ is $[5,6]$

$$
\begin{aligned}
T\left(x_{0}, 0\right)= & N\left(\frac{2 L^{2}}{D}\right)^{1 / 3}\left(\frac{x_{0}}{L}\right)^{1 / 6}\left(1-\frac{x_{0}}{L}\right)^{1 / 6}\left[F\left(1,-\frac{1}{3} ; \frac{7}{6} ; \frac{x_{0}}{L}\right)\right. \\
& \left.+F\left(1,-\frac{1}{3} ; \frac{7}{6} ; 1-\frac{x_{0}}{L}\right)\right]
\end{aligned}
$$

where $N=(4 / 3)^{1 / 6} / \Gamma(7 / 3)$, and $F(a, b ; c ; z)$ is the Gauss hypergeometric function [11].

Let us now obtain an approximate expression for $T(x, 0)$ using the method of Ref. [2]. In the case of white driving noise the Fokker-Planck equation (6) reads

$$
\frac{\partial p}{\partial t}=\frac{1}{2} D t^{2} \frac{\partial^{2} p}{\partial x^{2}}
$$

We will now solve this equation with the initial condition (7) and boundary conditions (8). Let us write the solution to Eq. (16) in the form of the following Fourier series:

$$
p\left(x, t \mid x_{0}, 0\right)=\sum_{n=1}^{\infty} a_{n}(t) \sin \left(\frac{n \pi x}{L}\right),
$$

that immediately satisfies the boundary conditions (8). Substituting Eq. (17) into Eq. (16) we see that the solution to (16) with initial condition (7) is

$$
p\left(x, t \mid x_{0}, 0\right)=\frac{2}{L} \sum_{n=1}^{\infty} e^{-n n^{2} \pi^{2} D t^{3} / 6 L^{2}} \sin \left(\frac{n \pi x_{0}}{L}\right) \sin \left(\frac{n \pi x}{L}\right)
$$

and the mean exit time reads [cf. Eq. (5)] 


$$
T\left(x_{0}, v_{0}=0\right)=M\left(\frac{L^{2}}{D}\right)^{1 / 3} \sum_{n=0}^{\infty} \frac{\sin \left[(2 n+1) \pi x_{0} / L\right]}{(2 n+1)^{5 / 3}}
$$

where $M=2^{7 / 3} \Gamma(1 / 3) / 3^{2 / 3} \pi^{5 / 3}$. This expression agrees with that of [2] in the white noise limit $\tau_{c} \rightarrow 0$. We note that Eq. (19) predicts the correct dependence on the length of the interval, that is, $T\left(x_{0}, 0\right) \sim L^{2 / 3}$ as $L \rightarrow \infty$ [cf. Eq. (15) and Ref. [5]]. In Fig. 1 we plot the (approximate) result (19) along with the exact result given by Eq. (15). We clearly see from this figure that the expression (19) is a poor approximation to the actual mean exit time. We therefore conclude that boundary conditions (8) are not suitable to deal with the escape problem for inertial processes.

This work has been supported in part by Dirección General de Investigación Científica y Técnica under Contract No. PB93-0812, by Societat Catalana de Física (Institut d'Estudis Catalans), by the U.S. Department of Energy through Grant No. DE-FG03-86ER13606, and by the Gaspar de Portolà Catalonian Studies Program at the University of California.
[1] J. Heinrichs, Phys. Rev. E 47, 3007 (1993).

[2] J. Heinrichs, Phys. Rev. E 48, 2397 (1993).

[3] J. Masoliver and K-G. Wang, Phys. Rev. E 51, 2987 (1995).

[4] In fact, Heinrichs evaluates $T\left(x_{0}\right)$ out of the interval $(-L, L)$. This case can be trivially reduced to that of $(0, L)$.

[5] J. Masoliver and J. M. Porrà, Phys. Rev. Lett. 75, 189 (1995).

[6] J. Masoliver and J. M. Porrà, Phys. Rev. E 53, 2243 (1996).

[7] M. C. Wang and G. E. Uhlenbeck, Rev. Mod. Phys. 17, 323
(1945).

[8] T. W. Marshall and E. J. Watson, J. Phys. A 18, 3531 (1985).

[9] J. M. Porrà, J. Masoliver, and K. Lindenberg, Phys. Rev. E 50, 1985 (1994).

[10] P. S. Hagan, C. R. Doering, and C. D. Levermore, SIAM J. Appl. Math. 49, 1480 (1989).

[11] Handbook of Mathematical Functions, edited by $\mathrm{M}$. Abramowitz and I. A. Stegun (Dover, New York, 1965). 